\title{
Prediction of Cardiovascular Clinical Outcomes with Novel Biomarker: the Focus on Growth Differentiation Factor 15
}

\author{
Alexander Berezin* \\ Professor, MD, PhD, Consultant of Therapeutic Unit, Internal Medicine Department, State Medical University \\ for Zaporozhye, 26, Mayakovsky av., Zaporozhye, Ukraine
}

*Corresponding Author: Alexander Berezin, Professor, MD, PhD, Consultant of Therapeutic Unit, Internal Medicine Department, State Medical University for Zaporozhye, 26, Mayakovsky av., Zaporozhye, Ukraine

\begin{abstract}
Heart failure (HF) is a major factor contributing to premature death in patients with established cardiovascular $(\mathrm{CV})$ disease. There is a large body of evidence that biological markers presumably natriuretic peptides, galectin-3, soluble ST2 and cardiac troponins could be the best tool for prediction of poor clinical outcomes in general population as well as in individuals with suspected or established CV diseases including HF. However, recent clinical trials have shown that abilities of these biomarkers regarding identification of morbidity and mortality risks distinguished in wide range and depended on numerous factors, i.e. age, sex, comorbidities, treatment regime and phenotypes of HF. Additionally, not all of these biomarkers except natriuretic peptides were best fitted to guided therapy of HF and consequently serial measures of them did not warrant improving of predictive value in follow-up especially in patients with preserved left ventricular ejection fraction. Growth-differentiation factor-15 (GDF-15) belongs to the transforming growth factor- $\beta$ superfamily that regulates mitochondrial function of wide range of cells that involve in inflammation, oxidative stress, apoptosis, immune reaction, fibrosis, reparation and malignancy. This mini review is depicted the possibilities to extrapolate the predictive capabilities of GDF-15 from metabolic and tumor diseases to CV diseases including HF.
\end{abstract}

Keywords: heart failure; cardiovascular disease; biomarkers; growth-differentiation factor-15; prognosis; clinical outcomes; predictive value.

\section{INTRODUCTION}

Heart failure is a leading cause of premature death in patients with established cardiovascular (CV) disease [1]. The prevalence of chronic HF regardless of left ventricular (LV) pump function increases worldwide that leads to shape hard economic burden of medical care of health systems in different countries [2]. In the developed countries the prevalence of chronic HF with reduced LV ejection fraction (EF) $<40 \%$ (HFrEF) especially due to ischemic and inflammatory reasons demonstrates a steadily decrease, while the prevalence of HF with preserved LVEF $=50-59 \%$ (HFpEF) in general population continues to progressively rise [3]. In contrast, in the developing countries new diagnosed cases of both phenotypes of chronic HF reveal a tendency to growth and associate with an increased risk of all-cause mortality and CV mortality [4]. The clinical presentation of chronic HF appears to be distinguished depending on age and a prevalence of the etiology causes including coronary artery disease (CAD), cardiomyopathies, myocarditis, diabetes mellitus, hypertension, chronic obstructive pulmonary disease etc. [5]. Although clinical entity of chronic HF with reduced LV ejection fraction (EF) $<40 \%$ (HFrEF) and preserved LVEF $=50-59 \%$ (HFpEF) respectively has defined, there is not completely clear whether HF (HFmrEF) related to mid-range LVEF=40-49\% could associate with clinical outcomes and prognosis similar other phenotypes of chronic HF [6]. The analysis of the TIME-CHF (Trial of Intensified versus standard Medical therapy in Elderly patients with Congestive Heart Failure) trail yielded that clinical status, profile of comorbidities including CAD and impaired quality of life of individuals with HFmrEF were comparable to patients with HFrEF, but these patients were not similar HFpEF in these fields [7]. In the Swedish Heart Failure Registry a higher adjusted risk of all-cause death and CV death in patients with HFmrEF and HFrEF versus HFpEF was defined [8]. Interestingly, there is overlapping in clinical and biomarker-based prediction of outcomes in different HF phenotypes [9, 10]. Indeed, natriuretic peptides (NPs) and cardiac troponins strongly 
predicted HFrEF and HFmrEF, but not HFpEF, although galectin-3 and soluble ST2 yielded much accurate predictive values for newly diagnosed HFpEF / HFmrEF and HFrEF-related admission to the hospital [7, 9]. Recent clinical trial has shown that biomarker-guided therapy based on serial measure of N-terminal pro-B-type NP (NT-proBNP) in comparison with standard HF therapy resulted in improved survival free of chronic HF admissions in HFrEF and HFmrEF, but not in HFpEF [7]. Therefore, there are several limitations regarding biomarkers' interpretation in chronic HF patients with older age, comorbidities and valsartan / sucubitrile use added to conventional HF therapy [11, 12]. Thus, novel biomarkers are required to be implemented to the clinical score aimed improving of predictive value of entire score system. Although growth-differentiation factor-15 (GDF15) is an established biomarker of inflammation, fibrosis and apoptosis, it took close discussion within recent several years as promising candidate to identify individuals at high risk of poor clinical outcomes. This mini review is depicted the possibilities to extrapolate the predictive capabilities of GDF-15 from metabolic and tumor diseases to $\mathrm{CV}$ diseases including HF.

\section{GROWTH-DIFFERENTIATION FACTOR-15: BIOLOGICAL ROLE AND FUNCTION}

GDF-15 (also known as macrophage inhibitory cytokine-1 - MIC-1, placental bone morphogenetic protein, nonsteroidal anti-inflammatory drug-activated protein-1, placental transforming growth factor-beta, prostate derived factor) belongs to the transforming growth factor- $\beta$ superfamily and was found an important regulator of mitochondrial function of wide range of cells that involve in the pathogenesis of immune reaction, inflammation, oxidative stress, fibrosis, reparation and malignancy [13]. In physiological and pathological conditions GDF-15 exhibited anti-inflammatory properties, which are produced by binding of this molecule with a currently unknown receptor [14]. There is evidence that GDF15 may specifically bind to an orphan member of the GDNF family receptor $\alpha$-like (GFRAL) and that intracellular signaling promoted by GDF15-GFRAL cooperation involve in the regulation of food intake by a central mechanism [15]. Over-expression of GDF-15 is up-regulated by several Th2-depended cytokines (interleukin [IL]-13, IL-4) via the Janus kinase-activated STAT6 pathway and metabolites (impaired fasting glucose, free radicals) through p53 and FoxM1-related intracellular signal system [16]. Thus, Th2-depended cytokines, which produced in white adipose tissue and perivascular adipose tissue, may regulate intra-adipose and systemic lipid and glucose metabolism via GDF-15 expression [17]. It has found that over-expression of GDF-15 leaded to lowered production of pro-inflammatory cytokines (IL-1beta, IL-6, IL-10, tumor necrosis factor [TNF]-alpha), various growth factors (fibroblast growth factor, insulin-like growth factor), active molecules (ICAM-1), as well as fibrotic mediators through a prevention of activation of Th1 cells. This effect is mediated by the Foxo3 and NFKB signaling pathways. Additionally, over-expression of GDF-15 reduced expression of phosphorylated RelA p65, pre-inflammatory and pro-apoptotic genes and thereby inhibited cell apoptosis and necrosis, as well as reduced mononuclear infiltration of tissues due to injury. Taking into consideration the fact regarding elevation of serum level of GDF-15 in various inflammatory and metabolic diseases they are considered GDF-15 could synthesize and secrete as a result in an adaptation to stress and inflammation via signaling pathways activated by mitochondrial stress [18].

Interestingly, there is evidence that the circulating GDF-15 produced by cardiac myocytes in turn acts on the liver cells to inhibit growth hormone signaling and thereby coordinates a cardiac function and growth / development of body that may have an important value in pediatric population with concomitant heart disease and failure to thrive [19]. Probably, GDF-15 could be a central autocrine/paracrine factor that plays a pivotal role in intercellular communication within the myocardium aimed preventing stress-induced, ischemia-induced and inflammatory-related cardiac diseases.

The essential role of GDF-15 in neovascularization and angiogenesis was confirmed in animal investigations and regarded an ability of the cytokine to promote the proliferation of human umbilical vein endothelial cells and remarkably improved vascular reparation [20]. In fact, GDF-15 acting as an angiogenic cytokine promoted tissue reparation in the healing and injury. In contrast, in cancer GDF15 acted as a promotor of antitumour immunity and revealed anti-inflammatory and immunosuppressive properties [16, 18]. The role of angiogenic ability of GDF-15 in metastatic malignancy is under investigation and requires to be scrutinized in details. Overall, GDF-15 appears to be a pleotropic cytokine, the role of which in pathogenesis of $\mathrm{CV}$, metabolic and inflammatory disease requires to be investigated in details. 


\section{GROWTH-DIFFERENTIATION FACTOR-15 IN INFLAMMATORY AND METABOLIC DISEASES}

Initially, GDF-15 is considered as an anti-inflammatory factor with reparative ability. Indeed, in diabetes mellitus, obesity and metabolic syndrome fasting serum levels of GDF-15 associated positively with some metabolic parameters (fasting glucose, glycated hemoglobin, HOMA index) and body mass index, age and men sex. In in nondiabetic patients GDF-15 correlated well with impaired fasting glucose and predicted an insulin resistance in general population [16]. Noted, GDF-15 is able to delay gastric emptying, modify a food preference, and regulate body energy intake and improve metabolic status through direct activation of central neurons in area postrema [18, 21]. The XENDOS (Xenical in the Prevention of Diabetes in Obese Subjects) trial was shown the interrelationship between the high levels of GDF15 in obese individuals and the risk of type 2 diabetes mellitus manifestation for 4 year follow-up period [22]. All these data clarify that GDF-15 could be a promising therapeutic target for the treatment of various diseases including obesity, diabetes mellitus and probably malignancy.

\section{ASSOCIATION BETWEEN GROWTH-DIFFERENTIATION FACTOR-15 AND CV DISEASES}

GDF-15 in elevated concentrations was found in numerous CV diseases, such as LV hypertrophy, stable CAD, myocardial infarction / acute coronary syndrome, acute and chronic HF, asymptomatic atherosclerosis [12, 23-25]. Moreover, GDF-15 has positively associated with LV mass, levels of IL-6 and matrix metalloproteinase (MMP)-9 levels [24]. In patients with pulmonary arterial hypertension secondary to congenital heart disease (CHD) serum levels of GDF-15 were significantly increased compared with CHD individuals without pulmonary arterial hypertension [25]. Authors reported that elevated plasma levels of GDF-15 positively associated with NYHA functional class, serum uric acid, NT-proBNP, pulmonary artery systolic pressure, mean pulmonary artery pressure, pulmonary blood flow, systemic blood flow and pulmonary vascular resistance, and as well as a lower mixed venous oxygen saturation [25]. It has been determined that the diagnostic value of NT-pro BNP and GDF-15 in turn of pulmonary arterial hypertension was similar. In atherosclerosis GDF-15 appears an ability to prevent ischemia and necrosis of cardiac cells, while a concentration of it in peripheral blood correlates well with cardiac fibrosis.

\section{GROWTH-DIFFERENTIATION FACTOR-15 IN CV PREDICTION}

GDF-15 was defined as good prognosticator of $\mathrm{CV}$ complications in patients with diabetes mellitus [26]. In the JUVENTAS (Rejuvenating Endothelial Progenitor Cells via Transcutaneous Intra-Arterial Supplementation) trial in patients with established peripheral artery disease elevated levels of GDF-15 were found a predictor of critical limb ischemia, increased risk of major amputation and all-cause mortality [27]. Elevated GDF-15 associated with a lack of reverse remodeling and increased mortality after transcatheter aortic valve replacement procedure and improves risk prediction of CV mortality rate adding to traditional score model [28]. It has found that are associated with an increased yearly rate of all-cause chronic obstructive pulmonary disease exacerbations in out-patients [29]. Because GDF-15 was found as an independent biomarker of all-cause mortality, CV death and non-fatal CV events in patients with $\mathrm{CAD}$ and atherosclerosis, it could support prescreening and selection high risk patients with non-ST-elevation acute coronary syndrome for early revascularization and aggressive medical therapy. Additionally, GDF-15 is considered as promising prognostic biomarker that predicts poor survival in not just individuals with chronic diseases, but in patients with critically illness including acute heart failure, sepsis, multiple organ failure [30-32].

\section{GROWTH-DIFFERENTIATION FACTOR-15 IN HF}

The HF-ACTION (Heart Failure: A Controlled Trial Investigating Outcomes of Exercise Training) study was yielded that GDF-15 in plasma positively associated with severity of HF, peak concentration of NT-proBNP, all-cause death rate and inversely related to peak oxygen uptake on cardiopulmonary exercise testing [21]. Interestingly, the serum levels of GDF-15 in patients with HFpEF were similar to those in HFrEF, while it associated with the severity of HF symptoms, echocardiographic parameters of LV dysfunction, 6 minute walk test distance and SF-36 physical score $[33,34]$. However, in HFpEF GDF-15 did not improve diagnostic discrimination when it is added to clinical status, cardiopulmonary exercise test findings and traditional biomarkers including high sensitive troponin T, galectin-3, soluble ST2 and NT-proBNP [35, 36]. In contrast, in HFrEF / HFmrEF diagnostic accuracy of GDF-15 was not inferior as that of NT-proBNP and combining both biomarkers may improve diagnostic discrimination $[36,37]$. Probably, multiple biomarkers including 
GDF-15 (i.e., high sensitive C-reactive protein + soluble ST2 \pm galectin-3 / NT-proBNP) added to novel score could predict HFpEF.

In chronic HF 8 month ivabradine therapy induced a reducing of GDF-15 levels by compared to baseline levels of the biomarker that associated with a significant reduction in the progress of LV remodeling [38]. Monitoring of serum levels of GDF-15 for follow-up period in HF individuals who are under treatment with strategy based on valsartan / sacubitril is not investigated. Therefore, GDF15 is independently associated with major bleeding in HF patients with atrial fibrillation receiving antithrombotic / antiplatelet therapies and this biomarker has included in novel bleeding risk score aimed improving decision making for risk stratification of the patients.

In conclusion: GDF-15 is elevated in numerous $\mathrm{CV}$ and metabolic diseases appearing to be a multifactorial cytokines with pro-angiogenic, anti-inflammatory and regenerative capacities. It is useful biomarker to stratify patients at highest CV risk and all-cause mortality risk. Additionally, elevated levels of GDF-15 may provide most robust prognostic information regarding distinguish of HFrEF / HFmrEF from HFpEF in addition to galectin-3 / soluble ST2 and / or NT-proBNP. The role of serial measured levels of GDF-15 in guided therapy of HF is not completely clear and requires to be investigated in large clinical trials.

\section{REFERENCES}

[1] Mozaffarian D, Benjamin EJ, Go AS, Arnett DK, Blaha MJ, Cushman M, et al; American Heart Association Statistics Committee and Stroke Statistics Subcommittee. Executive Summary: Heart Disease and Stroke Statistics-2016 Update: A Report from the American Heart Association. Circulation. 2016; 133(4): 447-54.

[2] Chioncel O, Lainscak M, Seferovic PM, Anker SD, Crespo-Leiro MG, Harjola VP, et al. Epidemiology and one-year outcomes in patients with chronic heart failure and preserved, mid-range and reduced ejection fraction: an analysis of the ESC Heart Failure Long-Term Registry. Eur J Heart Fail. 2017; 19(12):1574-1585.

[3] Guerra F, Brambatti M, Matassini MV, Capucci A. Current Therapeutic Options for Heart Failure in Elderly Patients. Biomed Res Int. 2017; 2017:1483873.

[4] Zhou HB, An DQ, Zhan Q, Liu ZH, Hua JH, Lai WY, et al. A retrospective analysis of clinical characteristics and outcomes of heart failure patients with different left ventricular ejection fractions. Zhonghua Nei Ke Za Zhi. 2017; 56(4): 253-257.

[5] Lupón J, Díez-López C, de Antonio M, Domingo M, Zamora E, Moliner P, et al. Recovered heart failure with reduced ejection fraction and outcomes: a prospective study. Eur J Heart Fail. 2017; 19(12):16151623.

[6] Farmakis D, Simitsis P, Bistola V, Triposkiadis F, Ikonomidis I, Katsanos S, et al. Acute heart failure with mid-range left ventricular ejection fraction: clinical profile, in-hospital management, and short-term outcome. Clin Res Cardiol. 2017; 106(5): 359-368.

[7] Rickenbacher P, Kaufmann BA, Maeder MT, Bernheim A, Goetschalckx K, Pfister O, et al; TIME-CHF Investigators. Heart failure with mid-range ejection fraction: a distinct clinical entity? Insights from the Trial of Intensified versus standard Medical therapy in Elderly patients with Congestive Heart Failure (TIME-CHF). Eur J Heart Fail. 2017; 19(12): 1586-1596.

[8] Koh AS, Tay WT, Teng THK, Vedin O, Benson L, Dahlstrom U, et al. A comprehensive population-based characterization of heart failure with mid-range ejection fraction. Eur J Heart Fail. 2017; 19(12): 16241634.

[9] Bhambhani V, Kizer JR, Lima JAC, van der Harst P, Bahrami H, Nayor M, et al. Predictors and outcomes of heart failure with mid-range ejection fraction. Eur J Heart Fail. 2017. doi: 10.1002/ejhf.1091. [Epub ahead of print]

[10] Pascual-Figal DA, Ferrero-Gregori A, Gomez-Otero I, Vazquez R, Delgado-Jimenez J, Alvarez-Garcia J, et al; MUSIC and REDINSCOR I research groups. Mid-range left ventricular ejection fraction: Clinical profile and cause of death in ambulatory patients with chronic heart failure. Int J Cardiol. 2017; 240: 265270.

[11] Berezin A, Kremzer A, Martovitskaya Y, Samura T, Berezina T. The novel biomarker risk prediction score in patients with chronic heart failure. Clinical Hypertension. 2016; 22 (3). doi: 10.1186/s40885-0160041-1

[12] Berezin AE. Prognostication in different heart failure phenotypes: the role of circulating biomarkers. Journal of Circulating Biomarkers. 2016; 5, 1-8 
[13] Peake BF, Eze SM, Yang L, Castellino RC, Nahta R. Growth differentiation factor 15 mediates epithelial mesenchymal transition and invasion of breast cancers through IGF-1R-FoxM1 signaling. Oncotarget. 2017; 8(55): 94393-94406.

[14] Breit SN, Johnen H, Cook AD, Tsai VW, Mohammad MG, Kuffner T, et al. The TGF-beta superfamily cytokine, MIC-1/GDF15: a pleotrophic cytokine with roles in inflammation, cancer and metabolism. Growth factors. 2011;29:187-195

[15] Yang L, Chang CC, Sun Z, Madsen D, Zhu H, Padkjær SB, et al. GFRAL is the receptor for GDF15 and is required for the anti-obesity effects of the ligand. Nat Med. 2017; 23(10):1158-1166.

[16] Hong JH, Chung HK, Park HY, Joung KH, Lee JH, Jung JG, et al. GDF15 Is a Novel Biomarker for Impaired Fasting Glucose. Diabetes \& metabolism journal. 2014;38:472-479

[17] Lee SE, Kang SG, Choi MJ, Jung SB, Ryu MJ, Chung HK, et al. Growth Differentiation Factor 15 Mediates Systemic Glucose Regulatory Action of T-Helper Type 2 Cytokines. Diabetes. 2017; 66(11): 2774-2788.

[18] Chung HK, Ryu D, Kim KS, Chang JY, Kim YK, Yi HSet al. Growth differentiation factor 15 is a myomitokine governing systemic energy homeostasis. The Journal of cell biology. 2017; 216:149-165.

[19] Wang T, Liu J, McDonald C, Lupino K, Zhai X, Wilkins BJ, et al. GDF15 is a heart-derived hormone that regulates body growth. EMBO Mol Med. 2017; 9(8):1150-1164.

[20] Wang S, Li M, Zhang W, Hua H, Wang N, Zhao J, et al. Growth differentiation factor 15 promotes blood vessel growth by stimulating cell cycle progression in repair of critical-sized calvarial defect. Sci Rep. 2017; 7(1): 9027.

[21] Xiong Y, Walker K, Min X, Hale C, Tran T, Komorowski R, et al. Long-acting MIC-1/GDF15 molecules to treat obesity: Evidence from mice to monkeys. Sci Transl Med. 2017; 9(412). doi: 10.1126/scitranslmed.aan8732.

[22] Kempf T, Guba-Quint A, Torgerson J, Magnone MC, Haefliger C, Bobadilla M, et al. Growth differentiation factor 15 predicts future insulin resistance and impaired glucose control in obese nondiabetic individuals: results from the XENDOS trial. Eur J Endocrinol. 2012;167:671-678

[23] Sharma A, Stevens SR, Lucas J, Fiuzat M, Adams KF, Whellan DJ, et al. Utility of Growth Differentiation Factor-15, A Marker of Oxidative Stress and Inflammation, in Chronic Heart Failure: Insights From the HF-ACTION Study. JACC Heart Fail. 2017; 5(10):724-734.

[24] Kou H, Jin X, Gao D, Ma R, Dong X, Wei J, et al. Association between growth differentiation factor 15 and left ventricular hypertrophy in hypertensive patients and healthy adults. Clin Exp Hypertens. 2017: 18. doi: 10.1080/10641963.2016.1273948. [Epub ahead of print]

[25] Li G, Li Y, Tan XQ, Jia P, Zhao J, Liu D, et al. Plasma Growth Differentiation Factor-15 is a Potential Biomarker for Pediatric Pulmonary Arterial Hypertension Associated with Congenital Heart Disease. Pediatr Cardiol. 2017; 38(8): 1620-1626.

[26] Berezin AE. Diabetes mellitus related biomarker: The predictive role of growth-differentiation factor- 15 . Diabetes \& Metabolic Syndrome: Clinical Research \& Reviews. 2016; 10 (1), S154-S157

[27] De Haan JJ, Haitjema S, den Ruijter HM, Pasterkamp G, de Borst GJ, Teraa M, et al. Growth Differentiation Factor 15 Is Associated With Major Amputation and Mortality in Patients With Peripheral Artery Disease. J Am Heart Assoc. 2017; 6(9). doi: 10.1161/JAHA.117.006225.

[28] Kim JB, Kobayashi Y, Moneghetti KJ, Brenner DA, O'Malley R, Schnittger I, et al. GDF-15 (Growth Differentiation Factor 15) Is Associated With Lack of Ventricular Recovery and Mortality After Transcatheter Aortic Valve Replacement. Circ Cardiovasc Interv. 2017; 10(12). doi: 10.1161/CIRCINTERVENTIONS.117.005594.

[29] Wu Q, Jiang D, Schaefer NR, Harmacek L, O'Connor BP, Eling T, et al. Over-production of growth differentiation factor 15 (GDF15) promotes human rhinovirus infection and virus-induced inflammation in the lung. Am J Physiol Lung Cell Mol Physiol. 2017. doi: 10.1152/ajplung.00324.2017. [Epub ahead of print]

[30] Buendgens L, Yagmur E, Bruensing J, Herbers U, Baeck C, Trautwein C, et al. Growth Differentiation Factor-15 Is a Predictor of Mortality in Critically Ill Patients with Sepsis. Dis Markers. 2017; 2017: 5271203.

[31] Falk M, Huhn R, Behmenburg F, Ritz-Timme S, Mayer F. Biomechanical stress in myocardial infarctions: can endothelin-1 and growth differentiation factor 15 serve as immunohistochemical markers? Int J Legal Med. 2017. doi: 10.1007/s00414-017-1726-z. [Epub ahead of print]

[32] Stahrenberg R, Edelmann F, Mende M, Kockskämper A, Düngen HD, Lüers C, et al. The novel biomarker growth differentiation factor 15 in heart failure with normal ejection fraction. Eur J Heart Fail. 2010; 12(12): 1309-16. 
Prediction of Cardiovascular Clinical Outcomes with Novel Biomarker: the Focus on Growth Differentiation Factor 15

[33] Berezin AE. Growth-Differentiation Factor-15 as Additional Prognostic Biomarkers in Heart Failure. Metabolomics. 2017; 7 (3): 194-195

[34] Jungbauer CG, Riedlinger J, Block D, Stadler S, Birner C, Buesing M, et al. Panel of emerging cardiac biomarkers contributes for prognosis rather than diagnosis in chronic heart failure. Biomark Med. 2014; 8(6): 777-89.

[35] Berezin AE. Biomarkers in heart failure. Journal of Blood \& Lymph. 2017; 7 (3): 172-179.

[36] Stahrenberg R, Edelmann F, Mende M, Kockskämper A, Düngen HD, Lüers C, et al. The novel biomarker growth differentiation factor 15 in heart failure with normal ejection fraction. Eur $\mathrm{J}$ Heart Fail. 2010;12(12):1309-1.

[37] Berezin AE. Up-to-date clinical approaches of biomarkers' use in heart failure. Biomed Res Ther 2017, 4(6): 1341-1370.

[38] Jirak P, Fejzic D, Paar V, Wernly B, Pistulli R, Rohm I, et al. Influences of Ivabradine treatment on serum levels of cardiac biomarkers sST2, GDF-15, suPAR and H-FABP in patients with chronic heart failure. Acta Pharmacol Sin. 2017. doi: 10.1038/aps.2017.167. [Epub ahead of print].

Citation: A. Berezin, "Prediction of Cardiovascular Clinical Outcomes with Novel Biomarker: the Focus on Growth Differentiation Factor 15", International Journal of Research Studies in Biosciences (IJRSB), vol. 6, no. 2, pp. 24-29, 2018. http://dx.doi.org/10.20431/2349-0365.0602004

Copyright: (c) 2018 Authors. This is an open-access article distributed under the terms of the Creative Commons Attribution License, which permits unrestricted use, distribution, and reproduction in any medium, provided the original author and source are credited. 